Check for updates

Cite this: Phys. Chem. Chem. Phys., 2019, 21, 3849 Accepted 15th January 2019

DOI: $10.1039 / c 8 c p 07060 a$

rsc.li/pccp
Received 15th November 2018,

\section{Atomistic insights into the dynamics of binary collisions between gaseous molecules and polycyclic aromatic hydrocarbon dimers $\dagger$}

\author{
Qian Mao, ${ }^{a}$ Juan Zhou, ${ }^{a}$ Kai H. Luo (D) *ab and Adri C. T. van Duin ${ }^{c}$
}

Polycyclic aromatic hydrocarbon (PAH) dimers are important intermediates in combustion and soot formation. The scattering dynamics of gaseous molecules colliding with PAH dimers and the subsequent PAH dimer stability are investigated by performing molecular dynamics (MD) simulations. Effects of properties of the surrounding gaseous molecules and PAH dimers as well as temperature are investigated in this study. Depending on the residence time of $\mathrm{N}_{2}$ molecules trapped by the PAH dimers, two scattering types, that is, specular scattering and inelastic scattering, have been observed, which is correlated to the temperature and the type of the PAH dimer. Specifically, specular scattering preferentially takes place at high temperatures on small PAH dimers, while inelastic scattering tends to happen at low temperatures on large PAH dimers. During collision, energy transfer between the gaseous molecule and the PAH dimer changes the thermodynamic stability of the PAH dimer. Statistical analysis indicates that the decomposition rate of a $\mathrm{PAH}$ dimer to $\mathrm{PAH}$ monomers is sensitive to temperature and the PAH dimer type. Furthermore, effects of the gaseous molecule type on the PAH dimer stability are considered. The molecular mass of the colliding gaseous molecule is a key factor in determining the $\mathrm{PAH}$ dimer stability, as heavier gaseous molecules are more effective in promoting the PAH dimer decomposition. Results from this study indicate that collisions with gaseous molecules decrease the $\mathrm{PAH}$ dimer stability, while increasing the PAH dimer size and decreasing the collision temperature both decrease the decomposition rate of the $\mathrm{PAH}$ dimer.

\section{Introduction}

Soot particles formed from gaseous molecules under oxygenstarved conditions at high temperatures contribute to air pollution and climate change. ${ }^{1}$ Substantial understanding has been gained about the soot formation mechanism in the following stages: aromatic formation, nucleation, coagulation and surface growth. ${ }^{2-6}$ Among these stages, the nucleation stage that describes the process from gaseous PAH monomers to condensed nascent soot particles is the least understood. Conceptually, soot nucleation is typically assumed to stem from polycyclic aromatic hydrocarbon (PAH) dimerization., ${ }^{4,7}$ The irreversible dimerization of PAH molecules is widely used

\footnotetext{
${ }^{a}$ Center for Combustion Energy, Key Laboratory for Thermal Science and Power Engineering of Ministry of Education, Department of Energy and Power Engineering, Tsinghua University, Beijing 100084, China

${ }^{b}$ Department of Mechanical Engineering, University College London, Torrington Place, London WC1E 7JE, UK. E-mail: K.Luo@ucl.ac.uk ${ }^{c}$ Department of Mechanical Engineering, The Pennsylvania State University, University Park, PA 16802, USA

$\dagger$ Electronic supplementary information (ESI) available: Parameter sets of ReaxFF force field for $\mathrm{C} / \mathrm{H} / \mathrm{O} / \mathrm{N}$ descriptions. See DOI: $10.1039 / \mathrm{c} 8 \mathrm{cp} 07060 \mathrm{a}$
}

to represent soot nucleation in soot modeling. ${ }^{8}$ However, both experimental studies in low-temperature supersonic flows ${ }^{9}$ and theoretical studies by molecular dynamics simulations ${ }^{10-12}$ indicate that the physical dimerization of pyrene only happens at low temperatures. Later, studies by Thomson and coworkers ${ }^{13,14}$ proposed a reversible dimerization model by considering the chemical and physical dimerization of PAHs, which is reported to have a better prediction of the soot volume fraction, average primary particle diameter, etc. The above two widely accepted soot nucleation models both neglect the impact from gaseous molecules, which are inevitable in experiments and under real combustion conditions. ${ }^{4,15}$

Collisions between soot nuclei and surrounding gaseous moelcules are quite common in combustion, which are also of great importance to particle formation especially at high pressures. ${ }^{16,17}$ Serving as the soot nuclei, PAH dimers should survive the collision from surrounding gaseous molecules and be stable enough to grow with other PAH monomers or dimers. As the collision time between $\mathrm{N}_{2}$ molecules in engines ( $1600 \mathrm{~K}$, 10 bar) is comparable to the PAH dimer lifetime, Schuetz and Frenklach ${ }^{18}$ stated that collisions with gaseous molecules is sufficient for PAH dimers to survive long enough for soot 
nucleation. Nonetheless, the effects of gaseous collisions on the PAH dimer stability have not yet been established with certainty. This results partly from the difficulty in taking experimental measurements with sufficient spatial and temporal resolutions to detect PAH dimers at low concentrations. From the aspect of the theoretical study or numerical modeling, the difficulties lie in the lack of a feasible force field description for interactions between gaseous molecules and PAHs in molecular dynamics (MD) simulations, and also in high computational costs of quantum chemistry (QC) calculations. Since higher peak pressures are becoming the norm in internal combustion engines, studying the interplay between the gaseous molecules and soot nuclei is ever more demanding. ${ }^{17,19}$

The objective of the present study is to provide a better understanding of the dynamics and kinetics of gaseous molecules colliding with PAH dimers. By performing a series of MD simulations, the influence of key factors, such as temperature, $\mathrm{PAH}$ dimer type, on the residence time and the scattering behavior of gaseous molecules is extensively examined. Furthermore, by quantifying the $\mathrm{PAH}$ dimer lifetime with and without gaseous molecule collisions, a kinetic model is developed with the aim to incorporate the key parameters that determine the PAH dimer stability.

\section{Methodology}

\subsection{Simulation method}

Binary collisions between gaseous molecules and PAH dimers are studied by MD simulations through tracing the movement of atoms. In the current study, a reactive force field (ReaxFF) is adopted, which includes most species in the periodic table. ${ }^{20}$ The ReaxFF MD can simulate both chemical reactions and physical dynamics in large systems with acceptable computational expense and accuracy. ${ }^{20}$ The computational cost for the single point energy calculation is several orders of magnitude lower than that of DFT calculations. ${ }^{9,11}$ In the ReaxFF, the energy calculation is composed of both bonded and nonbonded components based on the bond distance and bond order. $^{21}$ Parameters in the force field are trained against a series of QC calculations. ${ }^{22,23}$ The interactions between gaseous molecules, such as $\mathrm{N}_{2}, \mathrm{CO}, \mathrm{CO}_{2}$, and PAH dimers in the current study are described by the $\mathrm{C} / \mathrm{H} / \mathrm{O} / \mathrm{N}$ force field given in the ESI. $\dagger$ This is integrated from the ReaxFF C-2013 carbon parameters ${ }^{22}$ and parameters of the $\mathrm{C} / \mathrm{H} / \mathrm{O} / \mathrm{N}$ force field. ${ }^{23}$ This force field has been successfully used to study the thermodynamics and kinetics of PAHs and is reported to have a good description of the interactions between the gaseous molecules and PAHs. ${ }^{10,22,24-26}$

\subsection{Computational setup}

Before studying the binary collisions, the PAH dimer is firstly established. Our previous studies on PAH dimerization and soot inception have found that the PAH dimer in a stacked configuration is thermodynamically favored over the T-shaped one. ${ }^{10,24}$ Therefore, PAH dimers in stacked configurations are sampled evenly in time from binary collisions trajectories reported in our previous study of $\mathrm{PAH}$ dimerization. ${ }^{10}$ Fig. 1(a) shows the initial configuration of the binary collision with the PAH dimer placed in the center of a cubic box $\left(70 \times 70 \times 70 \AA^{3}\right)$. The initial distance between the $\mathrm{PAH}$ dimer and the gaseous molecule is $L_{\mathrm{d}}=50 \AA$, which is much larger than the distance of effective intermolecular interaction. The impact parameter $(b)$ is randomly selected from 0 to $\sqrt{\varepsilon d_{\text {dimer }}}$, where $\varepsilon$ is the enhancement factor, ${ }^{27}$ $d_{\text {dimer }}$ is the effective diameter of the PAH dimer expressed as,

$$
d_{\text {dimer }}=\sqrt{2} d_{\mathrm{PAH}}=2 d_{\mathrm{A}} \sqrt{\frac{n_{\mathrm{c}}}{3}},
$$

where $d_{\mathrm{A}}$ denotes the size of a single aromatic ring and equals $1.395 \sqrt{3} \AA$ and $n_{\mathrm{c}}$ is the number of carbon atoms in a PAH monomer. ${ }^{28}$ To achieve statistical significance, 1000 independent binary collision trajectories are studied at each temperature. The rotational energy $\left(E_{\text {rot }}\right)$ of the PAH dimer is randomly added in three dimensions according to the equipartition of energy. Relative translational velocities between the gaseous molecule and the $\mathrm{PAH}$ dimer are given to the gaseous molecules, which are in the Gaussian distribution of each temperature. In a real combustion system, collisions between gaseous molecules and PAH dimers happen at any orientation. Therefore, the collision angle $(\theta)$ is randomly sampled from the three dimensions. Gaseous molecules such as $\mathrm{N}_{2}, \mathrm{CO}, \mathrm{CO}_{2}$ are diatomic and polyatomic. The bonds in these molecules are treated as a harmonic oscillator. Fig. 1(b) summarizes the initial configurations of the 1000 independent binary collisions between $\mathrm{N}_{2}$ molecules and pyrene dimers at $400 \mathrm{~K}$. The arrows represent the collision orientations and relative collision velocities between gaseous molecules and the PAH dimer. The initial coordinate of the $\mathrm{PAH}$ dimer is the same for all the cases. MD simulations of the above binary collisions are performed adiabatically using a microcanonical ensemble (NVE) with a time step of 0.1 fs and last for $1 \times 10^{6}$ iterations, which accounts for a total simulation time of $100 \mathrm{ps}$. By solving the Newton's equations of motion with the ReaxFF, trajectories and velocities of the atom and molecules are given over time. MD simulations are performed using the large-scale atomic/molecular massively parallel simulator (LAMMPS) package ${ }^{29}$ implemented with reax/c. Snapshots and movies in this study are prepared by Ovito. $^{30}$

Experimental studies have indicated that the PAHs of naphthalene (A2), pyrene (A4) and coronene (A7) are typical precursors for soot inception. ${ }^{4}$ So PAH dimers formed from the (a)

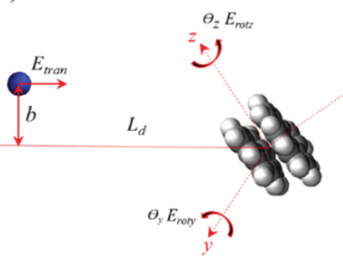

(b)

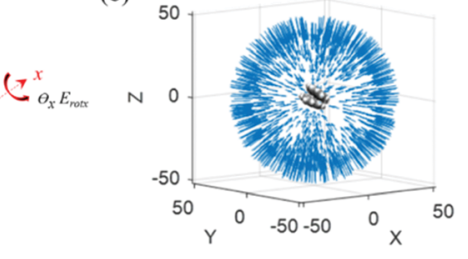

Fig. 1 (a) The initial configuration and setup for the binary collision between a gaseous molecule and a PAH dimer. (b) A summary of the initial configurations and the collision orientations of 1000 independent binary collisions between the gaseous molecule and the PAH dimer. 

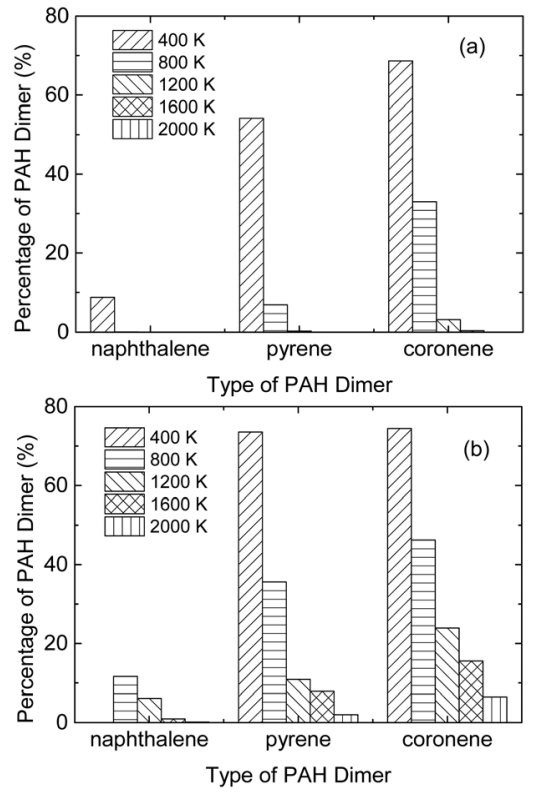

Fig. 2 Percentage of the PAH dimer with lifetime exceeding the mean collision time at (a) $1 \mathrm{~atm}$, (b) $10 \mathrm{~atm}$ and temperatures from 400 to $2000 \mathrm{~K}$.

above three types of PAH monomers are selected for the current study. The mean collision time $(\bar{t})$ between a gaseous molecule and $\mathrm{PAH}$ dimer is calculated from the mean free path $(\bar{\lambda})$ and the mean velocity $(\bar{\nu}),{ }^{31}$

$$
\bar{t}=\bar{\lambda} / \bar{v}=\frac{k_{\mathrm{B}} T}{\sqrt{2} \pi d^{2} P} / \sqrt{\frac{8 k_{\mathrm{B}} T}{\pi m}},
$$

where $k_{\mathrm{B}}$ is the Boltzmann constant, $T$ is the temperature, $P$ is the pressure, $m$ is the reduced mass expressed as $m=m_{\mathrm{dimer}} \cdot m_{\mathrm{gas}} l$ $\left(m_{\text {dimer }}+m_{\text {gas }}\right)$, and the collision diameter is $d=\left(d_{\text {dimer }}+d_{\text {gas }}\right) / 2$. Based on our previous study of PAH dimerization, ${ }^{10}$ percentage of the PAH dimer with lifetime exceeding the mean collision time between the $\mathrm{N}_{2}$ molecule and PAH dimer is presented in Fig. 2, which decreases with increasing temperature and decreasing dimer size. At $1 \mathrm{~atm}$, some coronene dimers survive longer than the mean collision time from 400 to $1600 \mathrm{~K}$. While for naphthalene, only when the temperature decreases to $400 \mathrm{~K}$, the dimer lifetime exceeds the mean collision time. Therefore, to reveal the scattering behavior of gas molecules and the PAH dimer stability, MD simulations are performed for PAH dimers whose lifetimes are longer than the mean collision time. Besides, effects of the combustion products, such as CO and $\mathrm{CO}_{2}$, are additionally considered in the current study and listed in Table 1.

\section{Results and discussion}

Based on the above simulation method and setup, Section 3.1 presents the dynamic trajectories of the collisions between the gaseous molecule and the PAH dimer at different temperatures. A quantitative study of the residence time and the scattering
Table 1 Overview of the initial settings for binary collisions

\begin{tabular}{|c|c|c|c|c|}
\hline Type & PAH dimer & $\begin{array}{l}\text { Gaseous } \\
\text { molecule }\end{array}$ & $\begin{array}{l}\text { Temperature } \\
\text { (K) }\end{array}$ & $\begin{array}{l}\text { No. of binary } \\
\text { collision (\#) }\end{array}$ \\
\hline 1 & Naphthalene & $\mathrm{N}_{2}$ & 400 & 1000 \\
\hline 2 & Naphthalene & $\mathrm{CO}$ & 400 & 1000 \\
\hline 3 & Naphthalene & $\mathrm{CO}_{2}$ & 400 & 1000 \\
\hline 4 & Pyrene & $\mathrm{N}_{2}$ & 400 & 1000 \\
\hline 5 & Pyrene & $\mathrm{N}_{2}$ & 600 & 1000 \\
\hline 6 & Coronene & $\mathrm{N}_{2}$ & 400 & 1000 \\
\hline 7 & Coronene & $\mathrm{N}_{2}$ & 600 & 1000 \\
\hline 8 & Coronene & $\mathrm{N}_{2}$ & 800 & 1000 \\
\hline 9 & Coronene & $\mathrm{N}_{2}$ & 1200 & 1000 \\
\hline 10 & Coronene & $\mathrm{N}_{2}$ & 1600 & 1000 \\
\hline 11 & Coronene & $\mathrm{CO}$ & 1600 & 1000 \\
\hline 12 & Coronene & $\mathrm{CO}_{2}$ & 1600 & 1000 \\
\hline
\end{tabular}

behavior of gaseous molecules on PAH dimers is provided in Section 3.2. And finally, Section 3.3 focuses on the effects of temperature, gaseous molecule type on the PAH dimer stability.

\subsection{Dynamics of binary collision}

Dynamics of gaseous molecules colliding with the PAH dimer and the subsequent dimer behaviors are investigated at different temperatures. Firstly, spatio-temporal evolutions of the binary collision events are monitored according to the molecule trajectories. The collision trajectories of a $\mathrm{N}_{2}$ molecule colliding with the coronene dimer with different collision velocities and orientations at $400 \mathrm{~K}$ are shown in Fig. 3(a and b), while those at $1600 \mathrm{~K}$ are shown in Fig. 3(c and d), respectively. In Fig. 3, the movement directions and the mass center trajectories of the $\mathrm{N}_{2}$ molecules and PAH dimers are indicated in the snapshots. Moreover, Fig. 4 shows the changes in the translational, kinetic, potential and total energies of the $\mathrm{N}_{2}$ molecules during collisions with PAH dimers shown in Fig. 3(b and d).

Firstly, the binary collision at a low temperature, e.g., $400 \mathrm{~K}$, is discussed. According to the collision trajectories shown in

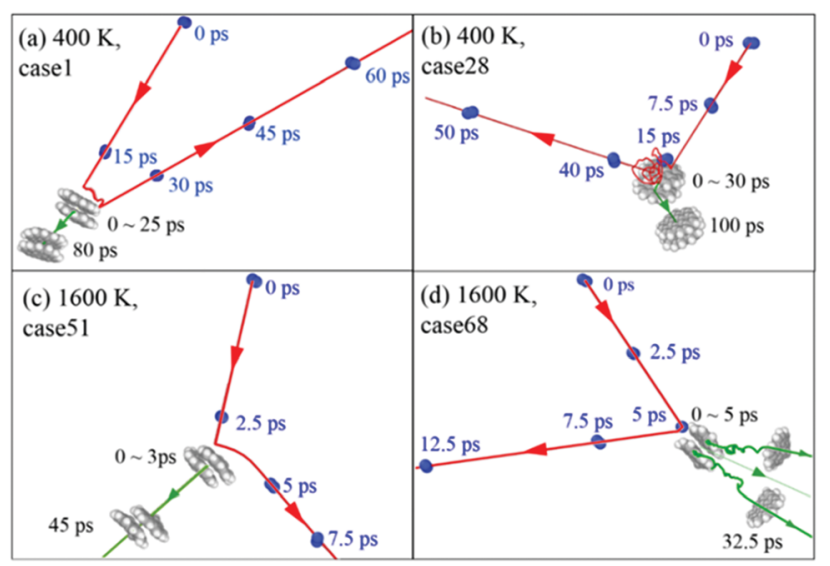

Fig. 3 Trajectories of the binary collision between the $\mathrm{N}_{2}$ molecule and the PAH dimer at varied temperatures ( $(a$ and b) $T=400 \mathrm{~K}$; ( $c$ and d) $T=1600 \mathrm{~K}$ ). The red and green lines represent the mass center trajectories of the $\mathrm{N}_{2}$ molecule and PAH dimer, respectively. Snapshots are recorded with the time evolution. Time and the position of $\mathrm{N}_{2}$ are recorded in blue and those of the $\mathrm{PAH}$ dimer or $\mathrm{PAH}$ monomers are in black. Blue, grey and white spheres represent nitrogen, carbon and hydrogen atoms. 

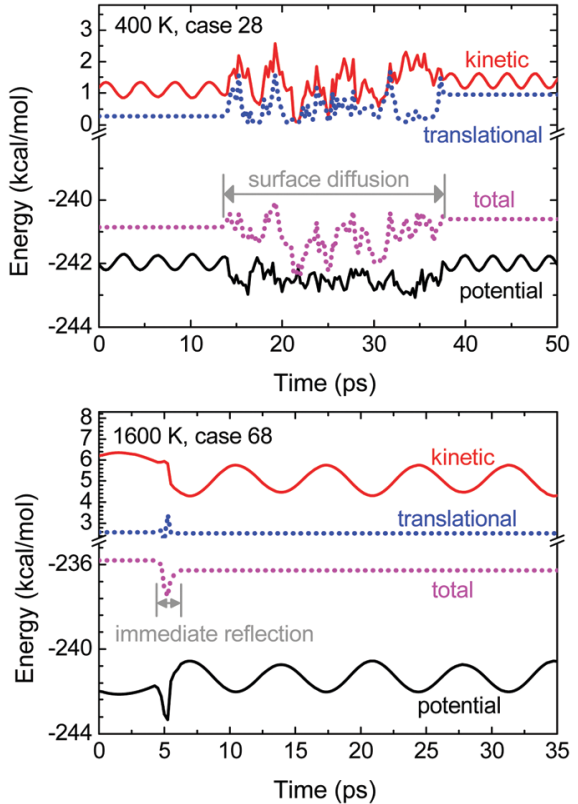

Fig. 4 Total, potential, kinetic and translational energy profiles of the colliding $\mathrm{N}_{2}$ molecules. The top and bottom panels show the results of the binary collisions of Fig. 3(b and d), respectively.

Fig. 3(b) and the energy evolutions in the upper panel of Fig. 4, the translational energy of the coronene dimer is zero before collision and the $\mathrm{N}_{2}$ molecule moves towards the coronene dimer with its initial translational velocity. Afterwards, the translational, kinetic and total energies of the $\mathrm{N}_{2}$ molecule increase upon collision, while the potential energy decreases slightly because of the intermolecular interactions between the $\mathrm{N}_{2}$ molecule and the PAH dimer. And then, the $\mathrm{N}_{2}$ molecule diffuses randomly on the PAH dimer surface as indicated by the irregular curved mass-centered trajectories in Fig. 3(b). After adsorption, the $\mathrm{N}_{2}$ molecule continuously exchanges energy with the PAH dimer as indicated in the fluctuations of the energy profiles. The diffusion duration of the $\mathrm{N}_{2}$ molecule on the $\mathrm{PAH}$ dimer is named the residence time, ${ }^{32}$ which is evaluated from the adsorption and desorption moments as demonstrated in Fig. 4. Although the initial translational collision velocity for the event shown in Fig. 3(a) is similar to that in Fig. 3(b), difference in the collision orientation leads to greatly different residence time of $\mathrm{N}_{2}$ molecules. That is, the residence is about $3.25 \mathrm{ps}$ for event (a) and as long as $21.25 \mathrm{ps}$ for event (b). Subsequently, the $\mathrm{N}_{2}$ molecule desorbs back from the PAH dimer through gaining energies from the PAH dimer as indicated by the increase in the total energy as well as the translational and kinetic energies. Afterwards, the $\mathrm{N}_{2}$ molecule travels in the gas phase at a constant translational energy. The periodic fluctuations of the kinetic and potential energy profiles in Fig. 4 result from the harmonic vibration of the $\mathrm{N}$ atom in the $\mathrm{N}_{2}$ molecule, that is, the transfer between the kinetic component and the potential component in the $\mathrm{N}_{2}$ molecule. Due to the momentum and the energy transfer during binary collision, the translational velocity of the PAH dimer is initiated after $\mathrm{N}_{2}$ collisions and the dimer travels as indicated by the mass center trajectories shown in green colour.
Compared to the binary collision at $400 \mathrm{~K}$, behaviors of the $\mathrm{N}_{2}$ molecule and PAH dimer at $1600 \mathrm{~K}$ are completely different as shown in Fig. 3(c and d). The energy profiles of the $\mathrm{N}_{2}$ molecule from binary collision shown in Fig. 3(d) are calculated and presented in the bottom panel of Fig. 4 . After collision, the translational and kinetic energies of the $\mathrm{N}_{2}$ molecule increase, while the total and potential energies decrease. The residence time of the $\mathrm{N}_{2}$ molecule on the PAH dimer surface is much shorter compared with that at low temperatures as it reflects back simultaneously from the PAH dimer after collision. According to the energy profiles, the translational energy of the $\mathrm{N}_{2}$ molecule after reflection almost remains the same as that before collision. The behavior of the $\mathrm{N}_{2}$ molecule in this case can be termed as specular scattering as it looks like rigid body collisions. ${ }^{16}$ Due to energy transfer between the $\mathrm{N}_{2}$ molecule and the PAH dimer after collision, the total energy of the $\mathrm{N}_{2}$ dimer is smaller than that before collision. This consequently leads to the increased total energy of the PAH dimer as the binary collision is performed in the NVE ensemble. Another interesting phenomenon is that the PAH dimer stability decreases after $\mathrm{N}_{2}$ collision as the PAH dimer decomposes into its monomers, which is indicated by the PAH trajectories shown in Fig. 3(d). Compared to the binary collision event shown in Fig. 3(c), it seems that the PAH dimer is much less stable when the $\mathrm{N}_{2}$ molecule collides on the inter-layer space between the PAH dimer than on the planar surface of the PAH dimer even at a lower collision energy. Similar behaviors of adsorption, reflection and even dissociation are commonly present in collision systems, such as the $\mathrm{Au}$ atom and $\mathrm{Au}$ cluster reported by Yang et al. ${ }^{17}$

\subsection{Gas-PAH dimer scattering}

As discussed in Section 3.1, temperature is an important factor in determining the scattering behaviors of the $\mathrm{N}_{2}$ molecule. Therefore, quantitative study on its relation with the temperature is performed in this section. The scattering behavior is classified by the residence time of the $\mathrm{N}_{2}$ molecule trapped in the PAH dimer after collision. Firstly, the temperature effect on the residence time of a $\mathrm{N}_{2}$ molecule is examined. Fig. 5 shows the percentage distributions of the residence time of $\mathrm{N}_{2}$ molecules on the coronene dimer from 400 to $1600 \mathrm{~K}$. Globally, the residence time distribution of the $\mathrm{N}_{2}$ molecule narrows with increasing temperature. That is, it ranges from 0 to more than $50 \mathrm{ps}$ at $400 \mathrm{~K}$, while it decreases to the range of $0-12.5 \mathrm{ps}$ at an elevated temperature of $1600 \mathrm{~K}$. According to Grančič et al., ${ }^{32}$ the residence time of $\mathrm{N}_{2}$ molecules on the soot particle in the first bar is regarded as specular scattering, which is not strictly specular but close to it as the collision trajectories show in Fig. 3(d). It is noticeable that the number of $\mathrm{N}_{2}$ molecules within the first bar increases with increasing temperature. This demonstrates that the $\mathrm{N}_{2}$ molecules are more likely to take specular scattering during binary collision as temperature increases. However, few $\mathrm{N}_{2}$ molecules can permanently get absorbed in the coronene dimer based on our current study from 400 to $1600 \mathrm{~K}$. The inelastic scattering is an intermediate state between the permanent absorption and the specular 


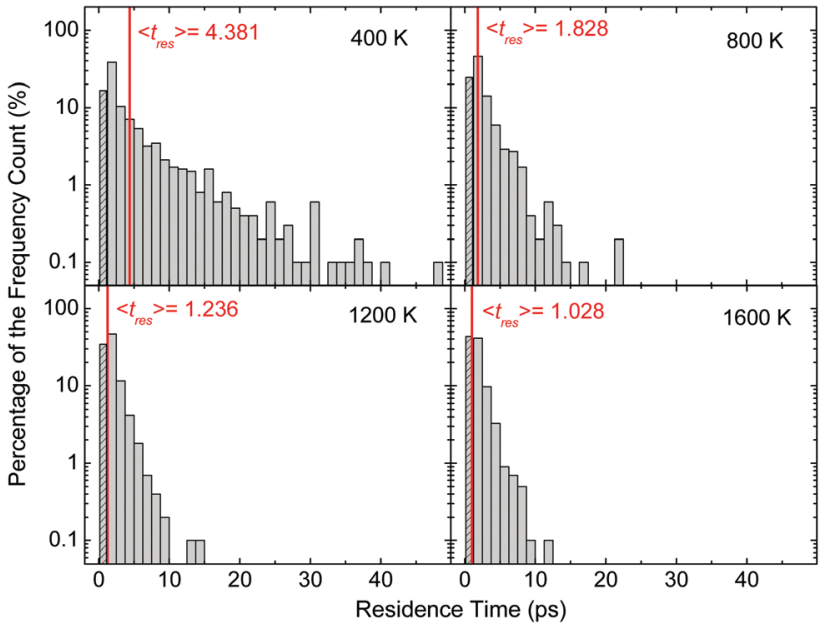

Fig. 5 Percentage distributions of the residence time of $N_{2}$ molecules with the coronene dimer at temperatures from 400 to $1600 \mathrm{~K}$.

scattering as presented in Fig. 3(a and b). Thus the percentage of the inelastic scattering can be regarded as the sum of the rest of the grey bars. Opposite to the percentage of specular scattering, that of the inelastic scattering decreases with increasing temperature. The mean residence time $\left(\left\langle t_{\text {res }}\right\rangle\right)$ of $\mathrm{N}_{2}$ molecules on the coronene dimer at each temperature is calculated and shown as the red line. Apparently, the mean residence time of $\mathrm{N}_{2}$ molecules on the coronene dimer is $4.381 \mathrm{ps}$ at $400 \mathrm{~K}$, and it decreases dramatically to $1.028 \mathrm{ps}$ as the temperature increases to $1600 \mathrm{~K}$. This means that the PAH dimer has higher adsorption ability at low temperatures, while it can hardly host the $\mathrm{N}_{2}$ molecules at high temperatures. Based on the energy profile shown in Fig. 4 , the behavior of $\mathrm{N}_{2}$ molecules in the PAH dimers can be quantified by $\Delta E_{\mathrm{pot}} / k_{\mathrm{b}} T{ }^{16}$ where $\Delta E_{\text {pot }}$ is the intermolecular interaction between the $\mathrm{N}_{2}$ molecule and the PAH dimer, and $k_{\mathrm{b}}$ is the Boltzmann constant. Since the system of the PAH dimer and the $\mathrm{N}_{2}$ molecule has higher thermal fluctuations at high temperatures, it prevents the adsorption of gaseous molecules. The behavior of the $\mathrm{N}_{2}$ molecule on the PAH dimer is similar to that of $\mathrm{N}_{2}$ collision with $\mathrm{Ag}$ clusters ${ }^{16}$ as the absorption tendency gets weaker at a higher temperature.

Now, the characteristic effects of the PAH dimer on the scattering behavior of the $\mathrm{N}_{2}$ molecule are investigated. Fig. 6 shows the percentage distribution of the residence time of $\mathrm{N}_{2}$ molecules on the naphthalene and pyrene dimers at $400 \mathrm{~K}$. Combined with the result of $\mathrm{N}_{2}$ molecules on the coronene dimer at $400 \mathrm{~K}$ in Fig. 5, it is found that the range of the residence time of $\mathrm{N}_{2}$ molecules is wider on the coronene dimer and it narrows with decreasing size of the PAH dimer. The mean residence time of $\mathrm{N}_{2}$ molecules on the PAH dimer decreases with increasing PAH dimer size. That is, the mean residence time decreases from $4.381 \mathrm{ps}$ on the coronene dimer to $2.738 \mathrm{ps}$ on the naphthalene dimer at $400 \mathrm{~K}$. This illustrates that a larger PAH dimer has higher possibility to trap the gaseous $\mathrm{N}_{2}$ molecules. Moreover, this also indicates that the percentage of the inelastic scattering of $\mathrm{N}_{2}$ molecules increases

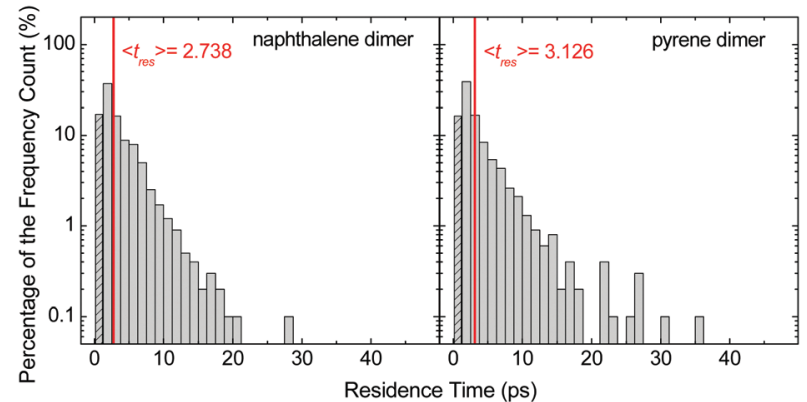

Fig. 6 Percentage distributions of the residence time of $\mathrm{N}_{2}$ molecule after colliding with naphthalene and pyrene dimers at the temperature of $400 \mathrm{~K}$.

with increasing PAH dimer size. The behavior of the $\mathrm{N}_{2}$ molecules on the PAH dimer agrees well with the $\mathrm{N}_{2}$ collision on $\mathrm{Ag}$ clusters as the specular-to-inelastic scattering transition shifts to larger particle sizes. ${ }^{16}$

\subsection{Stability of PAH dimers}

As demonstrated in Fig. 3, binary collisions between the $\mathrm{N}_{2}$ molecule and the PAH dimer lead to the decrease in the PAH dimer stability as some of the dimers fall apart into PAH monomers after $\mathrm{N}_{2}$ collisions. To quantify this effect, the lifetime of the PAH dimer after the gaseous molecule collision is evaluated. Specifically, the rate of PAH dimer decomposition after collision with a gaseous molecule is expressed by:

$$
\begin{gathered}
(\mathrm{PAH})_{2} \stackrel{\text { gas molecule }}{\longrightarrow} \mathrm{PAH}+\mathrm{PAH}, \\
R=\frac{\mathrm{d}\left[n_{\mathrm{dimer}}\right]}{\mathrm{d} t}=-k\left[n_{\mathrm{dimer}}\right],
\end{gathered}
$$

where $n_{\text {dimer }}$ is the number of the PAH dimers, $k$ is the rate of dimer decomposition after gaseous molecule collision. Relation between $n_{\text {dimer }}$ and its lifetime $t$ follows an exponential expression:

$$
\frac{n_{\text {dimer }}}{n_{0}}=\exp (-k \cdot t)
$$

where $n_{0}$ is the total number of PAH dimers before gaseous molecule collision. In order to explore the effects of gaseous molecule collision on the PAH dimer stability, the lifetime of the PAH dimer with and without gaseous molecule collision is compared by varying the gaseous molecule type, temperature and PAH dimer. Firstly, effects of temperature are investigated. Fig. 7 shows the ratio of the number of coronene dimers to the total number of gaseous molecule collisions $\left(n_{\mathrm{dimer}} / n_{0}\right)$ with respect to the dimer lifetime as well as the dimer decomposition rates at different temperatures. Apparently, the ratio of the coronene dimer after collisions with the $\mathrm{N}_{2}$ molecule at $400 \mathrm{~K}$ is mostly constant with its lifetime. This illustrates that no coronene dimer falls apart into monomers after $\mathrm{N}_{2}$ collisions within the simulation duration. As the temperature increases to $800 \mathrm{~K}$, the ratio of the coronene dimer $\left(n / n_{0}\right)$ decreases slightly with the lifetime. Then, it decreases much quicker when the temperature increases to $1200 \mathrm{~K}$ and even $1600 \mathrm{~K}$. The decomposition rate of 

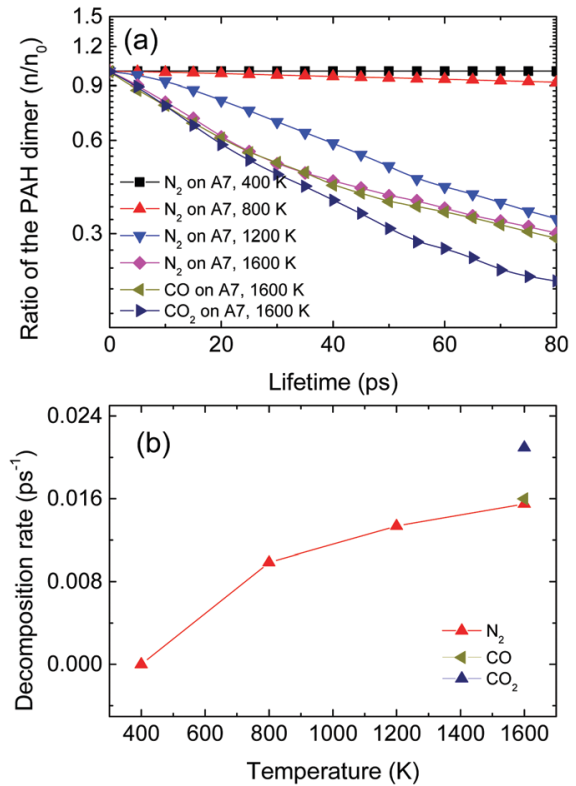

Fig. 7 (a) Ratio of the number of the coronene (A7) dimers to the total number of gaseous molecule collisions $\left(n / n_{0}\right)$, and (b) the decomposition rate of the coronene dimer under collisions with gaseous molecules $\left(\mathrm{N}_{2}, \mathrm{CO}\right.$ and $\left.\mathrm{CO}_{2}\right)$ from 400 to $1600 \mathrm{~K}$.

the PAH dimer into PAH monomers is extracted from the slope of the curve in Fig. 7(a) and illustrated in Fig. 7(b), which indicates that temperature has a significant impact on the stability of the $\mathrm{PAH}$ dimer at high temperatures. In addition, effects of the gaseous molecules of $\mathrm{CO}$ and $\mathrm{CO}_{2}$ on the coronene dimer stability at $1600 \mathrm{~K}$ are also studied and compared against that of $\mathrm{N}_{2}$ molecules in Fig. 7. It is found that the profile of the ratio of the coronene dimer to its lifetime after the collision with $\mathrm{CO}$ molecules mostly coincides with that of collision with $\mathrm{N}_{2}$ molecules, while that of collision with $\mathrm{CO}_{2}$ molecules is much quicker. This correspondingly means that the heavier the colliding gaseous molecule, the quicker the decomposition of the PAH dimer into PAH monomers.

Finally, the effect of the PAH dimer characteristic is studied at $400 \mathrm{~K}$ and the results of the ratios of the number of $\mathrm{PAH}$ dimers to the total number of binary collisions with respect to the lifetime are presented in Fig. 8. It is noteworthy that the $\mathrm{PAH}$ dimer stability after collision with gaseous molecules is sensitive to the PAH dimer characteristic. Specifically, for $\mathrm{N}_{2}$ collision with different $\mathrm{PAH}$ dimers, the decomposition rate of the PAH dimer decreases dramatically with increasing $\mathrm{PAH}$ size. That is, the coronene dimer is relatively more stable compared to the pyrene and naphthalene dimers after $\mathrm{N}_{2}$ collisions at the same temperature, which results from the stronger binding energy between coronene monomers. In addition, the effect of the gaseous molecule type is considered. It is quite similar to the results for coronene PAHs at $1600 \mathrm{~K}$ in Fig. 7 as heavier colliding gaseous molecules are more likely to break the naphthalene dimer into naphthalene monomers. According to our previous studies on the nascent soot morphology, ${ }^{24}$ it is found that above 400,600 and $800 \mathrm{~K}$, PAHs
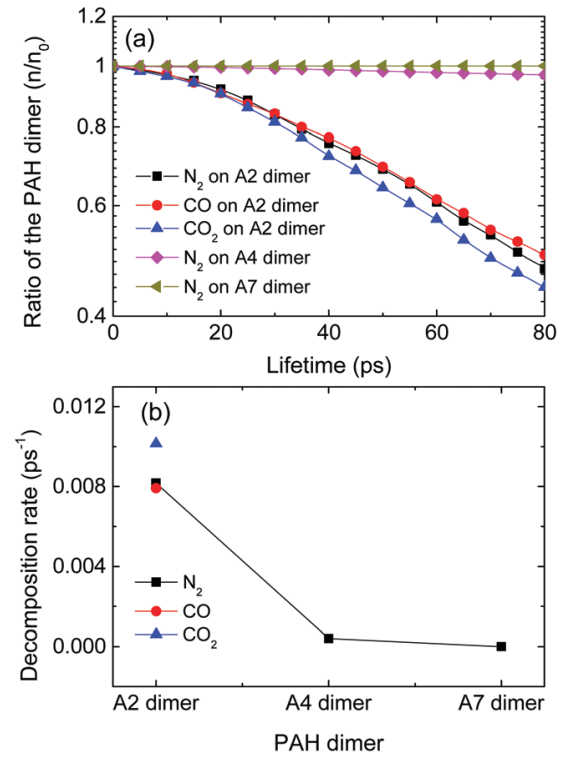

Fig. 8 (a) Ratio of the number of the surviving naphthalene, pyrene and coronene dimers to the total number of gaseous molecule collisions $\left(n / n_{0}\right)$, and (b) the decomposition rate of the PAH dimer, under collisions with gaseous molecules $\left(\mathrm{N}_{2}, \mathrm{CO}\right.$ and $\left.\mathrm{CO}_{2}\right)$ at $400 \mathrm{~K}$.

of naphthalene, pyrene and coronene cannot physically stack into nascent soot particles, respectively. Therefore, by integrating the effects of gaseous molecules, it can be concluded that gaseous molecule collisions may further decrease the possibility of $\mathrm{PAH}$ dimerization and formation of nascent soot particles in stacked configurations.

\section{Conclusions}

This paper presents a systematic study on the binary collision between the surrounding gaseous molecules and the PAH dimer by MD simulations. Key parameters, such as temperature, and properties of the PAH dimer and the gaseous molecule, are examined to clarify their influence on the scattering mechanism of the gaseous molecule on the PAH dimer and the PAH dimer stability. By analyzing the binary collision trajectory, the energy profile and the residence time of the gaseous molecule, it is found that the $\mathrm{N}_{2}$ molecules are more likely to result in specular scattering at elevated temperatures, while the possibility of inelastic scattering decreases as the temperature increases or the dimer size decreases. Moreover, few $\mathrm{N}_{2}$ molecules can become permanently absorbed on the coronene dimer even at $400 \mathrm{~K}$. Because of the energy transfer during the binary collision, the increase in the energy of the PAH dimer results in decreasing the PAH dimer stability compared to the situation without gaseous molecule collisions. The decomposition rate of the PAH dimer due to gaseous molecule collision is calculated and found to increase with increasing temperature. Moreover, larger PAH dimers are much more stable than smaller PAH dimers during collisions with $\mathrm{N}_{2}$ molecules. In addition to $\mathrm{N}_{2}$ molecules, the effect of the gaseous molecule type on the PAH dimer stability is also considered by including other molecules 
such as $\mathrm{CO}$ and $\mathrm{CO}_{2}$, which indicates that the mass of the colliding gaseous molecule is important for the $\mathrm{PAH}$ dimer stability. That is, $\mathrm{CO}_{2}$ has a higher possibility to lead to $\mathrm{PAH}$ dimer decomposition, and the effect of $\mathrm{CO}$ is quite similar to that of $\mathrm{N}_{2}$. The present study demonstrates that collisions with $\mathrm{N}_{2}$, CO or $\mathrm{CO}_{2}$ molecules lead to decreased stability of the PAH dimer, while increasing the size of the PAH dimer or decreasing the collision temperature tends to decrease the decomposition rate of the $\mathrm{PAH}$ dimer.

\section{Conflicts of interest}

There are no conflicts to declare.

\section{Acknowledgements}

Support from the National Natural Science Foundation of China (Grant No. 91441120 and 51390493) is gratefully acknowledged. Further support from the UK EPSRC under the project "UK Consortium on Mesoscale Engineering Sciences (UKCOMES)" (Grant No. EP/L00030X/1 and EP/R029598/1) is also acknowledged. The simulations were partly performed on the Tsinghua High-Performance Parallel Computer supported by the Tsinghua National Laboratory for Information Science and Technology.

\section{References}

1 H. A. Michelsen, Probing soot formation, chemical and physical evolution, and oxidation: a review of in situ diagnostic techniques and needs, Proc. Combust. Inst., 2017, 36, 717-735.

2 J. H. Miller, W. G. Mallard and K. C. Smyth, Intermolecular potential calculations for polycyclic aromatic hydrocarbons, J. Phys. Chem., 1984, 88, 4963-4970.

3 M. Frenklach, Reaction mechanism of soot formation in flames, Phys. Chem. Chem. Phys., 2002, 4, 2028-2037.

$4 \mathrm{H}$. Wang, Formation of nascent soot and other condensedphase materials in flames, Proc. Combust. Inst., 2011, 33, 41-67.

5 H. A. Michelsen, Probing soot formation, chemical and physical evolution, and oxidation: a review of in situ diagnostic techniques and needs, Proc. Combust. Inst., 2016, 000, 1-19.

6 K. Siegmann and K. Sattler, Formation mechanism for polycyclic aromatic hydrocarbons in methane flames, J. Chem. Phys., 2000, 112, 698-709.

7 B. Zhao, Z. Yang, M. V. Johnston, H. Wang, A. S. Wexler, M. Balthasar and M. Kraft, Measurement and numerical simulation of soot particle size distribution functions in a laminar premixed ethylene-oxygen-argon flame, Combust. Flame, 2003, 133, 173-188.

8 M. Frenklach and H. Wang, Detailed modeling of soot particle nucleation and growth, Symp. Combust., 1990, 23, 1559-1566.
9 H. Sabbah, L. Biennier, S. J. Klippenstein, I. R. Sims and B. R. Rowe, Exploring the role of PAHs in the formation of soot: pyrene dimerization, J. Phys. Chem. Lett., 2010, 1, 2962-2967.

10 Q. Mao, Y. Ren, K. H. Luo and A. C. T. van Duin, Dynamics and Kinetics of Reversible Homo-molecular Dimerization of Polycyclic Aromatic Hydrocarbons, J. Chem. Phys., 2017, 147, 244305.

11 S. H. Chung and A. Violi, Peri-condensed aromatics with aliphatic chains as key intermediates for the nucleation of aromatic hydrocarbons, Proc. Combust. Inst., 2011, 33, 693-700.

12 T. S. Totton, A. J. Misquitta and M. Kraft, A quantitative study of the clustering of polycyclic aromatic hydrocarbons at high temperatures, Phys. Chem. Chem. Phys., 2012, 14, 4081-4094.

13 N. A. Eaves, S. B. Dworkin and M. J. Thomson, The importance of reversibility in modeling soot nucleation and condensation processes, Proc. Combust. Inst., 2015, 35, 1787-1794.

14 M. R. Kholghy, N. A. Eaves, A. Veshkini and M. J. Thomson, The role of reactive $\mathrm{PAH}$ dimerization in reducing soot nucleation reversibility, Proc. Combust. Inst., 2018, 000, 1-9.

15 A. D. Abid, J. Camacho, D. A. Sheen and H. Wang, Quantitative measurement of soot particle size distribution in premixed flames-The burner-stabilized stagnation flame approach, Combust. Flame, 2009, 156, 1862-1870.

$16 \mathrm{Z}$. Li and H. Wang, Gas-nanoparticle scattering: a molecular view of momentum accommodation function, Phys. Rev. Lett., 2005, 95, 1-4.

17 H. Yang, E. Goudeli and C. J. Hogan, Condensation and dissociation rates for gas phase metal clusters from molecular dynamics trajectory calculations, J. Chem. Phys., 2018, 148, 164304.

18 C. A. Schuetz and M. Frenklach, Nucleation of soot: molecular dynamics simulations of pyrene dimerization, Proc. Combust. Inst., 2002, 29, 2307-2313.

19 A. L. Miller, C. B. Stipe, M. C. Habjan and G. G. Ahlstrand, Role of lubrication oil in particulate emissions from a hydrogen-powered internal combustion engine, Environ. Sci. Technol., 2007, 41, 6828-6835.

20 T. Senftle, S. Hong, M. M. Islam, S. B. Kylasa, Y. Zheng, Y. K. Shin, C. Junkermeier, R. Engel-Herbert, M. J. Janik, H. M. Aktulga, T. Verstraelen, A. Grama and A. C. T. van Duin, The ReaxFF reactive force-field: development, applications, and future directions, npj Comput. Mater., 2016, 2, 15011.

21 A. C. T. van Duin, S. Dasgupta, F. Lorant and W. A. Goddard III, ReaxFF: a reactive force field for hydrocarbons, J. Phys. Chem. A, 2001, 105, 9396-9409.

22 S. Goverapet Srinivasan, A. C. T. van Duin and P. Ganesh, Development of a ReaxFF potential for carbon condensed phases and its application to the thermal fragmentation of a large fullerene, J. Phys. Chem. A, 2015, 119, 571-580.

23 M. R. Weismiller, M. F. Russo, A. C. T. Van Duin and R. A. Yetter, Using molecular dynamics simulations with a ReaxFF reactive force field to develop a kinetic mechanism for ammonia borane oxidation, Proc. Combust. Inst., 2013, 34, 3489-3497. 
24 Q. Mao, A. C. T. van Duin and K. H. Luo, Formation of incipient soot particles from polycyclic aromatic hydrocarbons: a ReaxFF molecular dynamics study, Carbon, 2017, 121, 380-388.

25 K. Yoon, A. Rahnamoun, J. L. Swett, V. Iberi, D. A. Cullen, I. V. Vlassiouk, A. Belianinov, S. Jesse, X. Sang, O. S. Ovchinnikova, A. J. Rondinone, R. R. Unocic and A. C. T. van Duin, Atomisticscale simulations of defect formation in graphene under noble gas ion irradiation, ACS Nano, 2016, 10, 8376-8384.

26 Q. Mao and K. H. Luo, Trace metal assisted polycyclic aromatic hydrocarbons fragmentation, growth and soot nucleation, Proc. Combust. Inst., 2018, 000, 1-8.

27 S. J. Harris and I. M. Kennedy, The coagulation of soot particles with van der Waals forces, Combust. Sci. Technol., 1988, 59, 443-454.
28 A. Raj, M. Sander, V. Janardhanan and M. Kraft, A study on the coagulation of polycyclic aromatic hydrocarbon clusters to determine their collision efficiency, Combust. Flame, 2010, 157, 523-534.

29 S. Plimpton, Fast parallel algorithms for short-range molecular dynamics, J. Comput. Phys., 1995, 117, 1-19.

30 A. Stukowski, Visualization and analysis of atomistic simulation data with OVITO-the Open Visualization Tool, Modell. Simul. Mater. Sci. Eng., 2010, 18, 015012.

31 C. Law, Combustion physics, Cambridge University Press, 2006.

32 P. Grančič, J. W. Martin, D. Chen, S. Mosbach and M. Kraft, Can nascent soot particles burn from the inside?, Carbon, 2016, 109, 608-615. 